\title{
Regenerative Endodontic Treatment: A Case Report
}

\author{
${ }^{1}$ Dr Rasika Kashikar, ${ }^{2}$ Dr Manoj Chandak, ${ }^{3}$ Dr Prithwish Mukherjee, \\ ${ }^{4}$ Dr Aditya Patel \\ ${ }^{1}$ Post graduate student, Department of Conservative \\ Dentistry and Endodontics, Sharad Pawar Dental College, Sawangi Meghe Wardha Maharashtra \\ ${ }^{2}$ Professor and Head of Department, Sharad Pawar Dental College, Sawangi Meghe Wardha Maharashtra \\ ${ }^{3}$ Post graduate student, Department of Conservative Dentistry and Endodontics, Sharad Pawar Dental College, \\ Sawangi Meghe Wardha Maharashtra \\ ${ }^{4}$ Reader, Department of Conservative dentistry and Endodontics Sharad Pawar Dental College, \\ Sawangi Meghe Wardha Maharashtra
}

\begin{abstract}
Endodontic intervention in necrotic immature permanent teeth is usually a clinical challenge. With appropriate case selection, regenerative treatment can be effective, providing a desirable outcome. However, there is still no consensus on the optimal disinfection protocol or the method to achieve predictable clinical outcome. This article presents a case report of regenerative treatment in necrotic immature teeth, using mineral trioxide aggregate (MTA) as coronal barriers which led to successfull clinical outcomes.
\end{abstract}

Keywords: Regeneration; Endodontics; Dental Pulp Necrosis; Stem Cells

\section{Introduction}

Regenerative endodontic procedures (REPs) represent a paradigm shift for the treatment of necrotic pulps in immature permanent teeth, ranging from traditional barrier formation utilizing calcium hydroxide or mineral trioxide aggregate (MTA), to a biologically based treatment for root maturation Endodontic treatment of necrotic immature teeth is challenging. Proper preparation and obturation of the apical portion of immature teeth are difficult to achieve because of the thin, fragile dentinal walls and the blunderbuss anatomy [1]. Multiplesession apexification using calcium hydroxide used to be the treatment of choice for such cases [2]. Laterproposed one-step apexification by the induction of artificial barriers using materials such as MTA greatly decreased the frequency of sessions and duration of treatment [3]. However, both these techniques suffer a major limitation; they do not allow the continuation of root development and consequently result in the formation of a fragile root [4].

A recently suggested approach is based on creation of an environment that induces root maturation. This approach includes disinfection of the root canal system and use of antibiotic paste as an intracanal medicament. Revascularization is a biological alternative approach for treatment of immature necrotic teeth. In contrast to the conventional apexification and artificial formation of an apical barrier, revascularization enables root maturation [5,6]. Vital apical pulp tissue or Hertwig's epithelial root sheath might have remained in necrotic, open-apex teeth. If present, these tissues may proliferate and result in root development when the canal has been well disinfected and the inflammatory process has been reversed [7]

Modern era of regenerative endodontics was started by a case report by Banchs and Trope [8] in 2004. The proposed regenerative treatment generally starts with chemical Khoshkhounejadet. aldisinfection of the root canals. Different concentrations of sodium hypochlorite $(\mathrm{NaOCl})$ including $6 \%[9,10], 5.25 \%$ [5,8,11], $2.5 \%$ [12,13], and $1.25 \%$ [14] and different concentrations of chlorhexidine (CHX) including 2\% [9] and 0.12\% [11] have been successfully used for this purpose. The procedure continues by application of triple antibiotic paste as an intracanal medicament, which is composed of ciprofloxacin, metronidazole and minocycline, as suggested by Hoshino et al [15].

In the absence of clinical signs and symptoms of periradicular diseases, the treatment continues with removing the paste and inducing bleeding into the canals by irritating the periapical tissues. After formation of a blood clot, the orifice of the canal is sealed with MTA, which is placed over the clot as a biocompatible sealing material. Finally, the crown is permanently restored.This article describes revascularization treatment protocols in necrotic immature teeth, which led to successful Clinical outcome.

\section{Case Report}

A healthy 16 year-old male patient was referred to the Department of Endodontics at SharadPawar Dental College SawangiMegheWardha. The patient's chief complaint was fracture tooth in upper front region of jaw.The patient recalled a history of an impact trauma with \#21. Clinical examination revealed crown fracture with \#21 and history of root canal treatment with \#22. All the teeth in the maxillary anterior region were 
responsive to cold test, using electric heat pulp tester except for tooth \#21. There was no traceable sinus tract at the time. Radiography revealed immature root of tooth \#21 with a radiolucent periapical lesion. The diagnosis of necrotic pulp with asymptomatic apical periodontitis was made. The working length was determined and The root canal system was irrigated with $20 \mathrm{~mL}$ of $5.25 \% \mathrm{NaOCl}$ followed by $20 \mathrm{~mL}$ of $0.2 \% \mathrm{CHX}$ [8]. Triple antibiotic paste (ciprofloxacin, metronidazole, minocycline) in proportion of 1:1:1 was used as intracanal medicament for one week. In the next visit after 7 days, The antibiotic intracanal medicament was gently removed and flushed out of the canal with copious irrigation with 5.25\% $\mathrm{NaOCl}$. After drying the canal, bleeding was induced inside the canal with a sterile \#50 hand file (MANI Inc., Utsunomiya, Japan), which was inserted one millimeter beyond the apical foramen and the coronal part of the canal was sealed with ProRoot MTA (Dentsply) over the blood clot. A moist cotton pellet was placed over the MTA in the access cavity, and the tooth was temporarily restored with glass inomer cement.One week later, the patient was recalled to ensure the setting of MTA, and permanent restoration of the tooth was performed with composite resin (Dentsply) Three months later no signs or symptoms were recorded. At the six-month recall, narrowing of the canal,continuation of root formation was noted with healing of the radiolucent lesion on the radiographs.

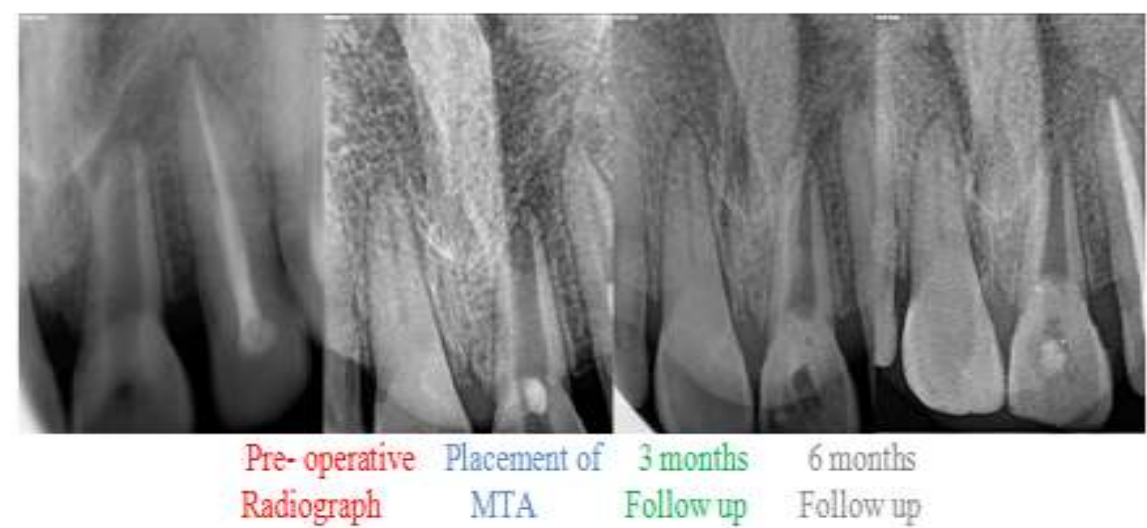

\section{Discussion}

In this case report, we evaluated a immature necrotic teeth with periapical radiolucencies. Pulp necrosis in this case was due to a traumatic event. Pulp revascularization was considered to be the treatment of choice in order to save the teeth and promote root development. The primary goal of regenerative endodontic procedures is healing of apical periodontitis as stated in the revised AAE guidelines (July 2013). According to the guidelines, the secondary goal is to increase root wall thickness and/or root length. The tertiary goal is to regain a positive response to pulp testing. Both the secondary and tertiary goals are desirable but possibly not essential to determine the clinical success. Root canal disinfection in regenerative endodontic treatment is challenging and debatable.One of the critical points is to choose the most effective antimicrobial agent for maximum cleaning of the root canal system. On the other hand, in teeth with an immature apex, the root canal is large in size, permitting easier permeation of antimicrobial agents into the root canal system and towards the periapical region.

In fact, the concern is how to prevent relatively toxic antimicrobial agents from gaining access to the periapical tissues, which may contain stem cells and vasculature that are necessary for the regeneration process. Attempts were made to find an antimicrobial agent with the least toxic effects in terms of both chemical composition and concentration. The use of Endo-Vac has been mentioned as a good strategy to avoid periapical extrusion of irrigants such as $\mathrm{NaOCl}[11]$.

The different concentrations of $\mathrm{NaOCl}$ including $6 \%[9,10], 5.25 \%[5,8,11], 2.5 \%[12,13]$ and $1.25 \%$ [14] and different concentrations of CHX $(0.12 \%$ to $2 \%)$ [9,15] have been successfully used for this purpose.In this case, the tooth was irrigated with $5.25 \% \mathrm{NaOCl}$ followed by $0.2 \% \mathrm{CHX}$ [8]. For removing the triple antibiotic paste in the second session, irrigation was performed using $5.25 \% \mathrm{NaOCl}$.

In the next visit, the antibiotic dressing was removed from the canal using normal saline. Next, $17 \%$ EDTA was used as the final irrigant because the use of EDTA is beneficial for providing dentin-derived growth factors [7].

The half- or full-strength (3\% and $6 \%$, respectively) concentrations of $\mathrm{NaOCl}$ have been shown to prevent stem cell attachment to dentin surfaces, and are toxic to stem cells of the apical papilla (SCAP) [4].

Previous studies showed that the use of 17\% EDTA significantly increased attachment of newly formed mineralized tissues to dentinal canal walls [5]. Growth factors are released from the root canal dentinal walls following EDTA irrigation. The success of revascularization/revitalization therapy depends on efficient 
disinfection of the root canal system. If infection persists in the root canal, not only regenerationButalso repair will not occur in the pulp-periapical tissue complex.

There may be a relation between time of trauma and quality of root development; the longer the duration of pulp necrosis, the lower the quality of root development after regenerative treatments [4]. Such association has also been discussed in previous studies reporting decreased or no root development and failure of the procedure [11].

Lenzi and Trope [2] discussed the possibility of longstanding infection destroying the cells capable of pulp regeneration. However, considering the successful outcomes of regenerative endodontic treatments in cases with long-lasting apical periodontitis, they concluded that this might not be the reason. Another possible explanation is the maturation of bacterial biofilm, which results in more difficult elimination by conventional protocols.

In this case bone formation was seen. The patient was asymptomatic. According to the AAE guidelines, healing of the apical periodontitis is the only essential requirement for clinical success [7]. However, in a more recent article, Fouad and Nosrat suggested that clinicians and the research community must reach a consensus about the clinically acceptable outcome. They suggested re-defining the clinical success and proposed that the clinical success must be re-defined as when calcification occurs in the absence of any signs and symptoms and the infection is completely resolved. Despite the fact that formation of intracanal mineralized tissue and pulp canal obliteration are inconsistent with the concept of regeneration, it seems logical to compromise the idealistic expectations in this field and overlook some of the shortcomings.

\section{Conclusion}

Regenerative endodontic procedures directly address the clinical need to treat immature teeth while fostering continued root development,demonstrating high rates of resolution of clinical signs and symptoms of pathosis.Combinations of antibiotics increases the success of revascularization therapy.

\section{References}

[1]. Khetarpal A, Chaudhary S, Talwar S, Ravi R, Verma M. Revascularization of immature permanent tooth with periapical lesion using a new biomaterial - A case report. Int J Dent Sci Res. 2013 Sep;1(1):20-2.

[2]. Rafter M. Apexification: a review. Dent Traumatol. 2005Feb;21(1)

[3]. Torabinejad M, Chivian N. Clinical applications of mineral trioxide aggregate. J Endod. 1999 Mar;25(3):197-205.

[4]. Nosrat A, Homayounfar N, Oloomi K. Drawbacks and unfavorable outcomes of regenerative endodontic treatments of necrotic

[5]. immature teeth: a literature review and report of a case. J Endod. 2012 Oct;38(10):1428-34.

[6]. Ding RY, Cheung GS, Chen J, Yin XZ, Wang QQ, Zhang CF. Pulp revascularization of immature teeth with apical periodontitis: a clinical study. J Endod. 2009 May;35(5):745-9.

[7]. Huang GT. A paradigm shift in endodontic management of immature teeth: conservation of stem cells for regeneration. J Dent. 2008 Jun;36(6):379-86.

[8]. Chen MY, Chen KL, Chen CA, Tayebaty F, Rosenberg PA, Lin LM. Responses of immature permanent teeth with infected necrotic pulp tissue and apical periodontitis/abscess to revascularization procedures. IntEndod J. 2012 Mar;45(3):294-305.

[9]. Banchs F, Trope M. Revascularization of immature permanent teeth with apical periodontitis: new treatment protocol? J Endod. 2004

[10]. Apr;30(4):196-200.

[11]. Reynolds K, Johnson JD, Cohenca N. Pulp revascularization of necrotic bilateral bicuspids using a modified novel technique to eliminate potential coronal discolouration: a case report. IntEndod J. 2009 Jan;42(1):84-92.

[12]. Shin SY, Albert JS, Mortman RE. One step pulp revascularization treatment of an immature permanent tooth with chronic apical abscess: a case report. IntEndod J. 2009 Dec;42(12):1118-26.

[13]. Petrino JA, Boda KK, Shambarger S, Bowles WR, McClanahan SB. Challenges in regenerative endodontics: a case series. J Endod. 2010 Mar;36(3):536-41.

[14]. Chueh LH, Huang GT. Immature teeth with periradicular periodontitis or abscess undergoing apexogenesis: a paradigm shift. J Endod. 2006 Dec;32(12):1205-13.

[15]. Chueh LH, Ho YC, Kuo TC, Lai WH, Chen YH, Chiang CP. Regenerative endodontic treatment for necrotic immature permanent teeth. J Endod. 2009 Feb;35(2): 160-4.

[16]. Thibodeau B, Trope M. Pulp revascularization of a necrotic infected

[17]. immature permanent tooth: case report and review of the literature. Pediatr Dent. 2007 Jan-Feb;29(1):47-50.

[18]. Hoshino E, Kurihara-Ando N, Sato I, Uematsu H, Sato M, Kota K, et al. In-vitro antibacterial susceptibility of bacteria taken from infected root dentine to a mixture of ciprofloxacin, metronidazole and minocycline. IntEndod J. 1996 Mar;29(2):125-30. 